\title{
ENSEÑANDO A LOS ALUMNOS EL MÉTODO CIENTÍfICO Y LA PUBLICACIÓN Y REVISIÓN POR PARES EN VETERINARIA: JOURNAL OF CORDOBA VETERINARY STUDENTS RESEARCH.
}

\section{JOURNAL OF CORDOBA VETERINARY STUDENTS RESEARCH: SCIENTIFIC METHOD AND RESEARCH PUBLICATION FOR VETERINARY STUDENTS}

\author{
Alejandro Perez-Ecija* \\ Francisco Javier Mendoza García \\ Antonio Buzón Cuervas \\ Laura Delgado Moreno \\ Alejandro.perez.ecija@uco.es (*Corresponding Author)
}

Universidad de Córdoba

\begin{abstract}
Veterinary students lack the necessary skills to correctly evaluate scientific papers. With this activity, the authors aimed to train the participants in the specific requirements of scientific writing and the research process in general. Groups of students designed and performed laboratory experiments (related to veterinary clinical pathology) and later they were asked to write a scientific paper and create a poster with their results. Papers were subjected to a scholarly peer review and later compiled in a publication (Journal of Cordoba Veterinary Students), while posters were defended in a students' congress. This activity was positively received by the students and participants were able to improve their critical thinking and scientific evaluation of veterinary literature.
\end{abstract}

Keywords: Journal, laboratory skills, research, veterinary.

\section{Resumen}

Los estudiantes de veterinaria comúnmente tienen un concepto de la investigación científica basado en prejuicios. El presente trabajo describe una actividad enfocada a fomentar el espíritu investigador en el alumnado de veterinaria y enseñar a los participantes el modus operandi que rige la publicación y difusión de cualquier trabajo científico. Para ello se crearon grupos de alumnos que desarrollaron un trabajo experimental relacionado con la patología clínica y diagnóstico laboratorial, siguiendo metodología científica válida. Tras ello, cada grupo preparó un manuscrito en inglés y un póster de sus resultados. El manuscrito se sometió a una revisión por pares anónima por parte de los propios alumnos y se publicó en formato journal, mientras que el póster se presentó en inglés en un congreso de estudiantes. La metodología descrita se ha demostrado suficientemente eficaz a la hora de establecer un espíritu crítico en la evaluación científica de los estudiantes participantes, fomentando al mismo tiempo su capacidad de innovación y emprendimiento.

Palabras clave: Investigación, journal, laboratorio, veterinaria.

\section{INTRODUCCIÓN}

Tras la reciente reestructuración de la docencia de Veterinaria en la Facultad de Córdoba en pos de implantar el nuevo Plan de Estudios del Grado de Veterinaria, y con el objetivo de una plena adaptación al Espacio Europeo de Educación Superior y superación de los requisitos exigidos para la acreditación europea de esta facultad (EAEVE), se ha hecho necesaria la realización de actividades que permitan al alumno adquirir competencias específicamente esenciales para su futura actividad clínica. Dichas actividades han de satisfacer una serie de aspectos que tradicionalmente estaban poco desarrollados en la docencia veterinaria y que, sin embargo, son centrales para el desarrollo completo del alumno como individuo con capacidad crítica científica y espíritu emprendedor.

Así, por ejemplo, conforme a la Orden ECI/333/2008, de 13 de febrero, por la que se establecen los requisitos para la verificación de los títulos universitarios oficiales que habiliten para el ejercicio de la profesión de Veterinario , entre las competencias que los estudiantes del Grado de Veterinaria han de adquirir se citan las siguientes:

-"Desarrollo de la práctica profesional con respeto a otros profesionales de la salud, adquiriendo habilidades relacionadas con el trabajo en equipo, con el uso eficiente de los recursos y en gestión de calidad".

-“Conocimiento y aplicación de técnicas diagnósticas complementarias y su interpretación”.

- "Biometría y estadística aplicados a las ciencias veterinarias".

- "Conocimiento y aplicación práctica de los principios y metodologías de la veterinaria".

Estas competencias son ampliamente trasversales y por ello están infrafomentadas en el actual Plan de estudios.

Por su parte, en el Libro Blanco de la Agencia Nacional de Evaluación de la Calidad Académica para la definición de competencias específicas en el grado de veterinaria, tras varias encuestas a los profesionales veterinarios clínicos, se registraron como habilidades con una formación deficitaria en la docencia universitaria las siguientes:

a) Capacidad para generar nuevas ideas.

b) Resolución de problemas.

c) Toma de decisiones. 
También en el Libro Blanco del Grado de Veterinaria (2005), se recoge, tras un estudio sobre inserción laboral en veterinaria, que prácticamente el cien por cien de los encuestados $(99,36 \%)$ consiguen un trabajo relacionado con la profesión. Esta misma conclusión se alcanzó en un trabajo realizado por FUNDECOR (Fundación Universitaria para el Desarrollo de la provincia de Córdoba), en colaboración con la Junta de Andalucía y la Universidad de Córdoba. En este último trabajo se concluyó que la mayoría de los egresados (75-80\%) de esta Universidad consiguieron su primer empleo en un periodo medio de 6 meses. De ambos estudios se podría deducir que la formación en veterinaria ha de ser al tiempo específica y completa, pues el alumno, al finalizar sus estudios, comienza a trabajar como profesional veterinario en un periodo de tiempo corto.

El campo de las publicaciones internacionales (los denominados journals y las revistas con alto índice de impacto) es totalmente desconocido para los alumnos de Veterinaria, que están mucho más acostumbrados a consultar libros recopilatorios didácticos y resúmenes bibliográficos en su lengua nativa. No obstante, nos encontramos ante la dicotomía de que las fuentes informativas internacionales con revisión con pares son las que mayor calidad científica aportan al lector, estando además dicha calidad informativa normalmente recogida en lengua inglesa. Ante la ausencia de formación y guía específica en este ámbito, muchos de los egresados y alumnos interesados en aumentar su horizonte lectivo han de aprender a discernir por sí mismos entre estas fuentes de información, (sin conocimientos previos sobre la metodología científica, los estudios estadísticos, los métodos típicos de investigación en biomedicina y la dinámica de la publicación científica y la revisión por pares).

Dados los problemas presentados y la idiosincrasia que la formación del método científico y la publicación en revistas por pares presenta en los alumnos de veterinaria (tenemos alumnos que han de comenzar a trabajar rápidamente una vez acaban sus estudios, que necesitan conocer y discernir adecuadamente las distintas fuentes de información pero que desconocen el modus operandi que existe tras el trabajo de publicación científica y revisión por pares), los autores nos planteamos aportar al alumno las distintas herramientas que le permitan desarrollar un espíritu crítico para evaluar distintas fuentes de información, inspirando al mismo tiempo en ellos el espíritu investigador y de revisión científica y promoviendo actitudes emprendedoras.

Además, todo este trabajo se diseñó de tal forma que se fomentasen también otras habilidades, como por ejemplo: trabajo en grupo y fomento de habilidades de liderazgo y reparto de trabajo; trabajo en lengua inglesa; uso de las nuevas tecnologías -TICs- y aplicación de conocimientos informáticos, edición de texto, bases de datos y hojas de cálculo.

$\mathrm{Al}$ estar sobradamente demostrado que, si estas competencias mencionadas se adquieren mediante actividades "hands on", las mismas se incorporan al acervo formativo del alumno mucho más rápida y eficazmente; los autores optamos por dotar a esta propuesta de un eminente carácter práctico. Para ello, dado que ambos son profesores clínicos con especial dedicación, formación y especialización en patología clínica y medicina interna veterinaria, siendo responsables de servicios en el Hospital Clínico Veterinario, y ambos tienen acceso a numerosas muestras tanto de animales sanos como con patología-, se propuso la realización por parte de los alumnos de todas las fases experimentales de forma práctica, evitándose en todo momento la necesidad de poner en riesgo a ningún animal de investigación. 


\section{OBJETIVOS}

Este proyecto tuvo como objetivo principal la adquisición por parte del alumno de veterinaria de habilidades y destrezas que le permitan afrontar con garantías su trabajo futuro, aprendiendo a discernir las fuentes de conocimiento e integrando el espíritu investigador, crítico y emprendedor en su acervo formativo.

Para actuar sobre las competencias citadas, se realizaron trabajos de investigación científica por parte de grupos de alumnos (trabajo en equipo), fomentándose el uso de nuevas tecnologías (editores de texto, bases de datos, programas de estadística biomédica, etc.). Dichos trabajos de investigación siguieron las directrices del método científico y acabaron redactándose en formato de papers, según la dinámica preponderante en la investigación biomédica y veterinaria (subdivisión en apartados típicos como título, abstract, introducción, material y métodos, resultados, conclusiones, referencias bibliográficas, tablas e imágenes). Tras escribir estos trabajos, los mismos se sometieron a una evaluación ciega por pares a cargo de los propios alumnos para finalmente proceder a la edición de un Journal con estos trabajos de investigación y defensa de los mismos de forma competitiva (en un formato tipo congreso científico).

Más específicamente, los objetivos concretos de esta acción fueron los siguientes:

- Fomentar la toma de decisiones críticas y el espíritu emprendedor.

- Capacitar al alumno para aplicar sus conocimientos teóricos en la práctica diaria.

- Alentar la sensación de responsabilidad del alumno frente a la profesión.

- Favorecer el desarrollo de la autocrítica y el juicio crítico justificado.

- Fomentar la capacidad de aportar ideas innovadoras y de calidad.

- Enseñar al alumno herramientas de diagnóstico laboratorial, metodología científica, bioestadística.

\section{METODOLOGÍA Y DESCRIPCIÓN.}

Para desarrollar la propuesta explicada, la actividad se dividió en diversos apartados. A continuación se detalla la metodología seguida en cada paso de la actividad, así como una descripción más detallada de la misma:

3.1.- REUNIÓN INFORMATIVA, CREACIÓN DE GRUPOS DE TRABAJO Y PROPUESTA DE TRABAJOS DE INVESTIGACIÓN. Se realizó una reunión informativa entre los alumnos objetivo de la propuesta (alumnos de Medicina Interna de $4^{\circ}$ de Grado de Veterinaria así como alumnos del Practicum de $5^{\circ}$ de Veterinaria; siendo en total -total de 125 alumnos en la reunión-). Tras explicar la dinámica de trabajo se crearon grupos randomizados de 4 a 5 alumnos y se les pidió una propuesta de trabajo de investigación relacionado con la Medicina Interna y Patología Clínica Veterinaria. Para fomentar el trabajo en equipo multidireccional y la capacidad de liderazgo, el reparto del trabajo entre cada componente fue organizado por los propios miembros del grupo.

\section{2.- ACEPTACIÓN DE LAS PROPUESTAS DE TRABAJO DE INVESTIGACIÓN.}

Los profesores responsables valoraron las propuestas de cada grupo de alumno. Como criterios de inclusión se tuvo en cuenta que se realizasen sobre muestras ó datos de pacientes y animales y que, por tanto, no requirieran una investigación biomédica activa (es decir, $\mathrm{NO}$ se aceptaron trabajos que requieran del paso por un Comité de Bioética). También se aceptó un trabajo que aprovechaba las muestras del control sanitario anual de los animales de prácticas propiedad del Hospital Clínico Veterinario Francisco Santisteban. En todos los casos se anonimizaron los datos de pacientes y clientes para el trabajo del alumnado. Se promovieron trabajos que requerían por parte del grupo de alumnos una actividad práctica además de un proceso de revisión bibliográfica. Los trabajos se centraron en técnicas de laboratorio (hematología, bioquímica, análisis de gases sanguíneos, hemostasia, realización de estudios citológicos, etc., etc.).

\section{3.- REALIZACIÓN PRÁCTICA DE LOS TRABAJOS DE INVESTIGACIÓN PROPUESTOS. EN}

En esta fase los alumnos usaron las instalaciones del Laboratorio del Dpto. De Medicina y Cirugía animal, así como el Laboratorio del HCV Fco. Santisteban. Se utilizaron fungibles específicos y equipamiento perteneciente a ambas instituciones. Durante la fase experimental los alumnos contaron con la supervisión y asesoramiento de los profesores responsables, en pos de poder culminar con éxito sus trabajos de investigación y comprobar su hipótesis experimental. Sin embargo, conviene recalcar que fueron los propios integrantes de cada grupo de trabajo los que decidieron el reparto de funciones. 


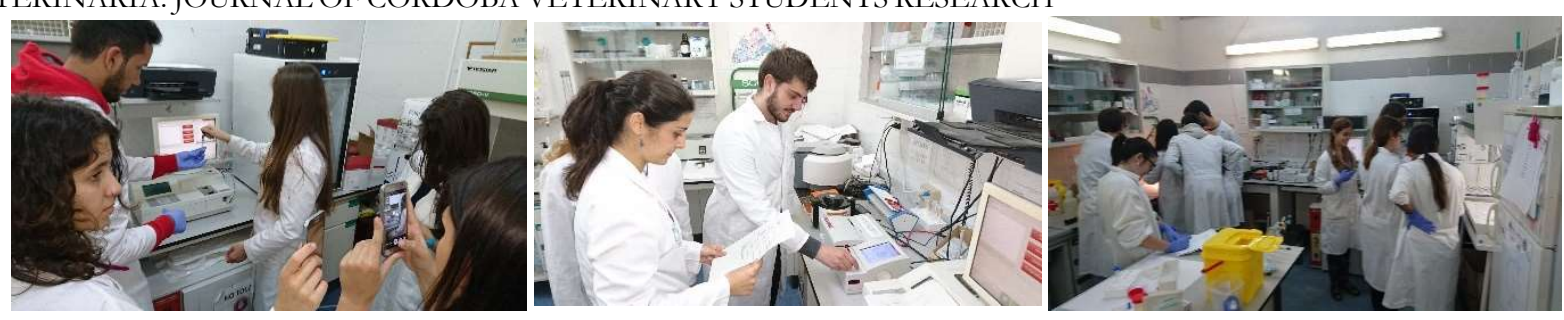

1. Fotografías de los alumnos participantes en la actividad durante la fase experimental de la misma.

\section{4.- ESCRITURA DE LOS TRABAJOS DE INVESTIGACIÓN.}

En esta fase cada grupo de alumnos escribió su paper con la típica estructura presente en biomedicina. De forma previa los profesores responsables ayudaron a los equipos mediante sesiones teóricas sobre la escritura científica y el procesado de datos. Los miembros del propio equipo tuvieron también que elegir el orden de autores de su trabajo.

\section{5.- REMISIÓN DE LOS TRABAJOS Y REVISIÓN CIEGA POR PARES.}

En esta fase los grupos remitieron sus trabajos de investigación en inglés y se realizó una revisión ciega por pares, siguiendo la dinámica existente en los journals de biomedicina. Los revisores pudieron requerir revisiones menores, mayores, aceptar ó denegar la publicación de los trabajos, siempre justificando sus decisiones de forma amplia y crítica. Todo este proceso se realizó igualmente en lenguaje inglés. En última instancia los profesores responsables actuaron como editores de la revista ó Journal.

\section{6.- PUBLICACIÓN DEL JOURNAL DE TRABAJOS DE INVESTIGACIÓN.}

Se realizó una publicación en papel, la cual se facilitó a cada participante en la actividad, con los trabajos aceptados, tras los cambios propuestos por los revisores y editores.

\section{7.- PREPARACIÓN DE LA DEFENSA ORAL DE LOS TRABAJOS.}

Siguiendo la dinámica existente en los congresos científicos nacionales e internacionales, cada grupo de alumnos seleccionó un ponente para la defensa del trabajo. Siempre trabajando en grupo, realizaron un póster según las instrucciones típicas existentes en congresos biomédicos, donde brevemente expusieron su trabajo de investigación. El ponente defendió y el póster ante un jurado que pudo realizar preguntas y precisiones sobre el tema de investigación.

\section{8.- SELECCIÓN DE TRABAJOS GANADORES Y ENTREGA DE PREMIOS.}

Como una forma de fomentar la participación en esta actividad, así como para aumentar el grado de emprendimiento e implicación científica en las fases anteriores, el jurado seleccionó el trabajo ganador en función de lo expuesto en los journals así como la defensa oral realizada ante los pósters. El material que se generó en esta fase quedó en exposición pública para su consulta por parte de otros alumnos de veterinaria en las instalaciones del Hospital Clínico Veterinario y Dpto. de Medicina y Cirugía animal.

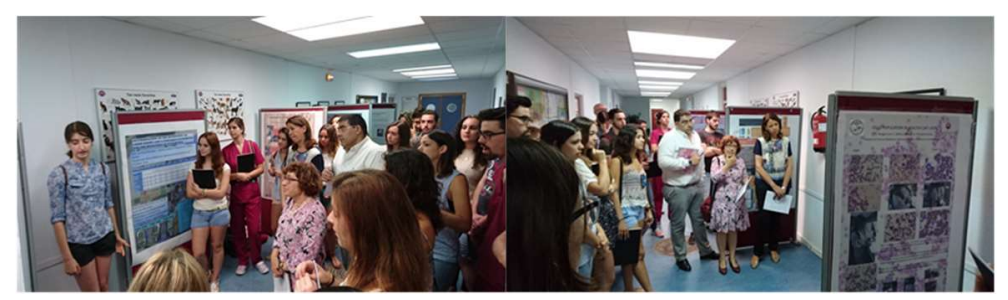

2. Varias imágenes de la sesión de defensa de los trabajos de investigación, con los alumnos participantes explicando sus experimentos y resultados al jurado del Congreso.

\section{RESULTADOS OBTENIDOS}

En este proyecto han participado 65 alumnos de cuarto y quinto curso del Grado de Veterinaria (actividad voluntaria), en grupos con un número máximo de 5-6 componentes. Al final de la actividad se les pidió a los participantes que valorasen la actividad. Todos los participantes contestaron la encuesta final de evaluación de la actividad.

Los resultados arrojaron una valoración muy positiva de la misma (media: 9.2/10) por parte del alumnado.

Al utilizarse un proceso de revisión doble ciego, la evaluación aumentó su objetividad, con varios ejemplos de revisiones mayores e incluso un rechazo. A cada revisor se le asignó una serie de parámetros base (calidad de la investigación, adecuación del formato, calidad de la bibliografía, calidad de la expresión escrita, calidad de los 
gráficos y tablas) que evaluar y se le pidió una decisión final (aceptación, revisión mayor, revisión menor o rechazo). En cada una de estas revisiones los profesores actuaron como editores, guardando en todo momento la confidencialidad de revisores y autores e incluyendo a sucesivos revisores cuando se producían divergencias en el criterio de los dos primeros alumnos.

La mayoría de trabajos fueron aceptados $(6 / 13 ; 46 \%)$, con 3 revisiones menores $(23 \%$, todas ellas por fallos en el formato), 3 revisiones mayores $(23 \%, 2$ por baja calidad de la bibliografía y 1 por baja calidad de la expresión escrita) y 1 rechazo $(7 \%)$. La tasa de coincidencia entrerevisores fue del $85 \%$, siendo las divergencias encontradas entre el grado de revisión (mayor/menor).
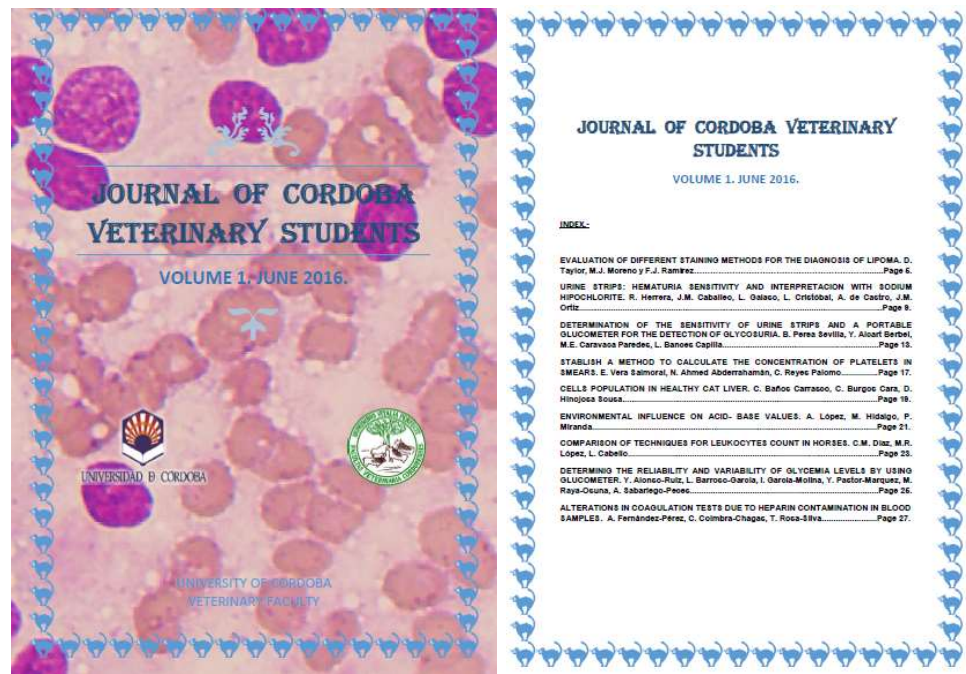

3.- Portada y parte del índice del Journal of Cordoba Veterinary Students Volume 1, en el cual se recogieron todos los trabajos realizados por los alumnos participantes sobre las experiencias laboratoriales que completaron.

\section{UTILIDAD/ANÁLISIS}

La experiencia ha causado una gran aceptación y la participación ha sido amplia y entusiasta. En el curso 2016/2017, los solicitantes hemos pedido la ampliación, mediante esta misma convocatoria, de este proyecto en pos de poder expandir el mismo a otros estudiantes, tanto de otros cursos de Veterinaria como, sobre todo, de otros Grados relacionados con las Ciencias básicas y de la Salud. Este nuevo proyecto ha sido aceptado por la Universidad de Córdoba, encontrándose actualmente en ejecución.

Los resultados obtenidos por las investigaciones de los alumnos han sido recogidos en una publicación en papel (Journal of Cordoba Veterinary students VOLUME 1. JUNE 2016) que se facilitó a todos los participantes, al jurado del congreso científico y al resto de alumnos de dicho año interesados.

Los resultados de este trabajo han sido igualmente difundidos mediante presentación de los mismos en el III Congreso VetDoc de Docencia en Veterinaria, celebrado en Córdoba en junio de 2016 y publicación en la revista VetDoc, en el número extraordinario de 2016.- http://www.vetdoc.es/index.php?journal=vetdoc.

\section{CONCLUSIONES}

Como conclusión, los autores pueden confirmar la buena acogida de la actividad y su utilidad para el desarrollo y fomento de habilidades y competencias transversales usualmente poco afrontadas en el Grado de Veterinaria. La metodología detallada se demostró eficaz a la hora de instaurar un ambiente de trabajo idóneo para la investigación aplicada a la clínica veterinaria entre los alumnos de veterinaria, creándose grupos con gran dinamismo e intercambio de ideas e hipótesis de trabajo. La realización "hands-on" de la parte experimental resultó de especial interés para los participantes, que pudieron comprobar en primera instancia las diferencias entre los resultados reales y los esperables en el ámbito investigador y pudieron igualmente poner en práctica el método científico, conociendo su idiosincrasia y problemática.

Por su parte, al enfrentarse al sistema de revisión por pares y la estructuración típica de las publicaciones científicas, los estudiantes fueron conscientes del nivel de exigencia para la difusión de trabajos científicos de primer nivel, generando un espíritu crítico hacia distintas fuentes de información que difícilmente se puede hacer nacer de otra forma. 


\section{BIBLIOGRAFÍA}

BOE (2008). ORDEN ECI/333/2008, de 13 de febrero, por la que se establecen los requisitos para la verificación de los títulos universitarios oficiales que habiliten para el ejercicio de la profesión de Veterinario. Boletín Oficial del Estado núm. 40, de 15 de febrero de 2008, paginas 8355-8357.

Libro Blanco de la Agencia Nacional de Evaluación de la Calidad Académica para la definición de competencias específicas en grado el de veterinaria. Disponible http://www.aneca.es/var/media/150400/libroblanco jun05 veterinaria.pdf.

RUIZ ABAD, L. La profesión veterinaria en el siglo XXI: un estudio de mercado, Madrid, 2001.

Informe de Emprendedores e Inserción Laboral de los Egresados de la Universidad de Córdoba $2007-2008$. FUNDECOR, Universidad de Córdoba y Consejería de Empleo de la Junta de Andalucía. 